\title{
NEW APPLICATIONS OF \\ THE BIEDENHARN-TEMPLE OPERATOR $\left({ }^{1}\right)$
}

\author{
P. A. HORVÁTHY \\ Département de Mathématiques, Université, \\ Parc de Grandmont, F-37200 TOURS (France) \\ e-mail: horvathy@univ-tours.fr
}

\begin{abstract}
.
The Biedenharn approach to the Dirac-Coulomb problem is applied to a system considered by D'Hoker and Vinet, which consists of a spin $\frac{1}{2}$ particle in the combined field of a Dirac monopole plus a $\lambda^{2} / r^{2}$ potential. The explicit solution is obtained by diagonalizing the 'Biedenharn-Temple operator', $\Gamma$.
\end{abstract}

\section{Introduction.}

In a paper anticipating supersymmetric quantum mechanics, [1], Biedenharn proposed a new approach to the Dirac-Coulomb problem. He starts with the square of the Dirac equation. This equation can be written in a non-relativistic Coulomb form (but with a fractional angular momentum), by using the 'Biedenharn-Temple' $[1,2]$ operator $\Gamma$. The solutions of the first-order equation can be recovered from those of the second-order equation by projection.

Recently [3], we have applied Biedenharn's method to the 'dyon' system of D'Hoker and Vinet [4]. Here we illustrate the Biedenharn method on yet another example (also considered by D'Hoker and Vinet $[5])$, namely that of a spin $\frac{1}{2}$ particle described by the four-component Hamiltonian

$$
H=\left(\begin{array}{ll}
H_{1} & \\
& H_{0}
\end{array}\right)=\frac{1}{2}\left\{\boldsymbol{\pi}^{2}-q \frac{\boldsymbol{\sigma} \cdot \hat{\mathbf{r}}}{r^{2}}+\frac{\lambda^{2}}{r^{2}}-\lambda \gamma^{5} \frac{\boldsymbol{\sigma} \cdot \hat{\mathbf{r}}}{r^{2}}\right\}
$$

where $\lambda$ is a real constant.

\section{The Biedenharn-Temple operator for a monopole system.}

The Hamiltonian (1.1) can be viewed as associated to a static gauge field on $\mathbf{R}^{4}, \mathbf{A}=q \mathbf{A}_{D}, A_{4}=\lambda / r$, where $\mathbf{A}_{D}$ denotes the vector potential of a Dirac monopole of unit strength. The square of the associated Dirac operator,

$$
\not D:=\left(\begin{array}{ll}
Q & Q^{\dagger}
\end{array}\right)=\left(\begin{array}{cc}
\boldsymbol{\sigma} \cdot \boldsymbol{\pi}-i \frac{\lambda}{r} \\
\boldsymbol{\sigma} . \boldsymbol{\pi}+i \frac{\lambda}{r} &
\end{array}\right),
$$

is seen to be (1.1). Since the Dirac operator is chiral-supersymmetric in any even dimension, the partner hamiltonians have the same spectra.

The conserved total angular momentum is $\mathbf{J}=\mathbf{L}+\boldsymbol{\sigma} / 2$, where $\mathbf{L}=\ell-q \hat{\mathbf{r}}, \ell=\mathbf{r} \times \boldsymbol{\pi}$. The eigenvalues of $\mathbf{J}^{2}$ are $j(j+1), j=q-1 / 2, q+1 / 2, \ldots$. Set

$$
w=\boldsymbol{\sigma} . \hat{\mathbf{r}}, \quad z=\boldsymbol{\sigma} \cdot \ell+1 \quad \text { and } \quad \mathcal{K}=-\left(\begin{array}{cc}
i z \\
i z
\end{array}\right) .
$$

Note that $w^{2}=1$, and that $z$ anticommutes with $w$ and $\boldsymbol{\sigma} . \boldsymbol{\pi}$,

$$
\{z, w\}=0 \quad \text { and } \quad\{z, \boldsymbol{\sigma} . \boldsymbol{\pi}\}=0 .
$$

$\left({ }^{1}\right)$ Dedicated to Prof. L. C. Biedenharn, on his 70th birthday. Festschrift in honor of L. C. Biedenharn, ed. B. Gruber. Plenum : New York (1994). 
$\mathcal{K}$ commutes with the Dirac operator $\not \supset$. Using $(\boldsymbol{\sigma} . \mathbf{L})^{2}=\mathbf{L}^{2}+i \boldsymbol{\sigma}(\mathbf{L} \times \mathbf{L})=\mathbf{L}^{2}-\boldsymbol{\sigma} . \mathbf{L}$, one proves that $\mathcal{K}^{2}=z^{2}=\mathbf{J}^{2}+1 / 4-q^{2}$. Thus, $z$ (and $\mathcal{K}$ ) have irrational eigenvalues,

$$
\kappa= \pm \sqrt{(j+1 / 2)^{2}-q^{2}} .
$$

Since $j \geq q-1 / 2, \mathcal{K}$ is hermitian, but for $j=q-1 / 2$ its eigenvalue $\kappa$ vanishes and $\mathcal{K}$ is not invertible.

Using $\boldsymbol{\sigma} \cdot \boldsymbol{\pi}=-i w\left(\partial_{r}+1 / r-z / r\right)$, the supercharges $Q$ and $Q^{\dagger}$ can be written as

$$
\left\{\begin{aligned}
Q=-i w\left(\partial_{r}+\frac{1}{r}-\frac{y}{r}\right) & =-i\left(\partial_{r}+\frac{1}{r}+\frac{x}{r}\right) w \\
Q^{\dagger}=-i w\left(\partial_{r}+\frac{1}{r}-\frac{x}{r}\right) & =-i\left(\partial_{r}+\frac{1}{r}+\frac{y}{r}\right) w
\end{aligned}\right.
$$

where we introduced the notations

$$
x=z-\lambda w \quad \text { and } \quad y=z+\lambda w .
$$

$(x w=-w y)$. Let us define the Biedenharn-Temple operator as

$$
\Gamma=-\left(\boldsymbol{\sigma} \cdot \ell+1+\gamma^{5} \lambda w\right) \quad \text { i.e. } \quad-\left(z+\gamma^{5} \lambda w\right) \equiv-\left(\begin{array}{cc}
y & \\
& x
\end{array}\right) .
$$

Since $\Gamma^{2}=z^{2}+\lambda^{2}=\mathbf{J}^{2}+1 / 4+\lambda^{2}-q^{2}$, the eigenvalues of $\Gamma$ are

$$
\gamma= \pm \sqrt{(j+1 / 2)^{2}+\lambda^{2}-q^{2}}, \quad \operatorname{sign} \gamma=\operatorname{sign} \kappa .
$$

In terms of $\Gamma, \not{ }^{2}$ becomes

$$
\not D^{2}=\left(\begin{array}{cc}
Q^{\dagger} Q & \\
& Q Q^{\dagger}
\end{array}\right)=-\left(\partial_{r}+\frac{1}{r}\right)^{2}+\frac{\Gamma(\Gamma+1)}{r^{2}} .
$$

Although $\Gamma$ does not commute with $\not D$, it is conserved for the quadratic dynamics.

\section{The explicit solution.}

The operator $\Gamma$ can be diagonalized generalizing the procedure in Ref. 6 . Let us first assume that $j \geq j+1 / 2$, and let $L_{ \pm}:=j \pm 1 / 2$ be the orbital angular momentum quantum number. Consider first the two-component spinors

$$
\varphi_{ \pm}^{\mu}=\sqrt{\frac{L_{ \pm}+1 / 2 \pm \mu}{2 L_{ \pm}+1}} Y_{L_{ \pm}}^{\mu-1 / 2}\left(\begin{array}{l}
1 \\
0
\end{array}\right) \pm \sqrt{\frac{L_{ \pm}+1 / 2 \mp \mu}{2 L_{ \pm}+1}} Y_{L_{ \pm}}^{\mu+1 / 2}\left(\begin{array}{l}
0 \\
1
\end{array}\right),
$$

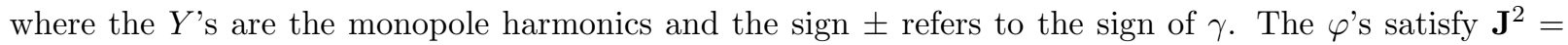
$j(j+1), J_{3}=\mu,(\mu=-j, \cdots, j), \mathbf{L}^{2}=L_{ \pm}\left(L_{ \pm}+1\right)$. The 2-spinors

$$
\left\{\begin{array}{l}
\chi_{+}=\frac{1}{2 j+1}\left(\begin{array}{cc}
\varphi_{+} & +\frac{q}{j+1 / 2+|\kappa|} \varphi_{-}
\end{array}\right) \\
\chi_{-}=\frac{1}{2 j+1}\left(-\frac{q}{j+1 / 2+|\kappa|} \varphi_{+}+\varphi_{-}\right)
\end{array}\right.
$$

satisfy the important relations

$$
z \chi_{ \pm}= \pm|\kappa| \chi_{ \pm} \quad \text { and } \quad w \chi_{ \pm}=\chi_{\mp},
$$

as well as $\mathbf{J}^{2}=j(j+1), J_{3}=\mu,(\mu=-j, \cdots, j)$. Hence

$$
\left\{\begin{array}{l}
\phi_{+}=\left(|\kappa|+j+\frac{1}{2}\right) \chi_{+}-\lambda \chi_{-}, \quad \phi_{-}=\lambda \chi_{+}+\left(|\kappa|+j+\frac{1}{2}\right) \chi_{-} \\
\Phi_{+}=\left(|\kappa|+j+\frac{1}{2}\right) \chi_{+}+\lambda \chi_{-}, \quad \Phi_{-}=-\lambda \chi_{+}+\left(|\kappa|+j+\frac{1}{2}\right) \chi_{-}
\end{array}\right.
$$


diagonalize $x$ and $y$,

$$
x \phi_{ \pm}^{\mu}=\mp|\gamma| \phi_{ \pm}^{\mu} \quad \text { and } \quad y \Phi_{ \pm}^{\mu}=\mp|\gamma| \Phi_{ \pm}^{\mu} .
$$

The operator $w=\boldsymbol{\sigma} \cdot \hat{\mathbf{r}}$ interchanges the $x$ and $y$ eigenspinors,

$$
w \phi_{ \pm}^{\mu}=\Phi_{\mp}^{\mu} .
$$

For $j=q-1 / 2$, no $\varphi_{-}$is available and $\chi_{-}$is hence missing. $\chi_{+}$is proportional to the lowest $\varphi_{+}$in (3.1). Thus, there are no $\phi_{-}$-states in the $\gamma^{5}=-1$ sector and no $\Phi_{+}$states in the $\gamma_{5}=1$ sector. However, in each $\gamma^{5}$ sector, $(3.2)$ yields $(2 q)$ states, namely

$$
\phi_{+}^{\mu 0}=\Phi_{-}^{\mu 0} \propto \sqrt{\frac{q+1 / 2 \pm \mu}{2 q+1}} Y_{q}^{\mu-1 / 2}\left(\begin{array}{l}
1 \\
0
\end{array}\right) \pm \sqrt{\frac{q+1 / 2 \mp \mu}{2 q+1}} Y_{q}^{\mu+1 / 2}\left(\begin{array}{l}
0 \\
1
\end{array}\right)
$$

They are (+1)-eigenstates of $w$.

The eigenfunctions of $\not D^{2}$ are then found as

$$
\begin{aligned}
& \left\{\begin{array} { l } 
{ \Psi _ { \pm | \gamma | } = u _ { \pm } ( \begin{array} { c } 
{ \Phi _ { \pm } } \\
{ 0 }
\end{array} ) } \\
{ \psi _ { \pm | \gamma | } = u _ { \pm } ( \begin{array} { c } 
{ 0 } \\
{ \phi _ { \pm } }
\end{array} ) }
\end{array} \quad \text { for } \left\{\begin{array}{cc}
\gamma^{5}=1 \\
\\
\gamma^{5}=-1
\end{array}\right.\right. \\
& \left\{\begin{array}{l}
\Psi_{-}^{0}=u_{-}^{0}\left(\begin{array}{c}
\Phi_{-} \\
0
\end{array}\right) \\
\psi_{+}^{0}=u_{+}^{0}\left(\begin{array}{c}
0 \\
\phi_{+}
\end{array}\right)
\end{array} \quad \text { for } j \geq q+1 / 2\right.
\end{aligned}
$$

Thus, the radial functions $u_{ \pm}(r)$ solve the equations

$$
\left[-\left(\partial_{r}+\frac{1}{r}\right)^{2}+\frac{\gamma(\gamma+1)}{r^{2}}-2 E\right] u_{ \pm}=0
$$

This is the wave equation for a free particle except for the fractional 'angular momentum' $\gamma$. Its solutions is hence given by the Bessel functions,

$$
u_{ \pm}(r) \propto r^{-1 / 2} J_{|\gamma| \mp \frac{1}{2}}(\sqrt{2 E} r) .
$$

For $\lambda=0$ we recover the formulae in [6]. The well-known self-adjointness problem in the $j=q-1 / 2$ sector shows up in that the eigenvalue $\gamma$ in Eq. (2.8) vanishes in this case. (Self-adjointness of $\not{ }^{2}$ requires in fact $|\lambda| \geq 3 / 2[5])$.

Another particular value is $\lambda= \pm q$, when the Biedenharn-Temple operator has half-integer eigenvalues,

$$
\gamma= \pm\left(j+\frac{1}{2}\right) .
$$

In this case, $\gamma(\gamma+1)$ is the same for $-|\gamma|$ as for $|\gamma|-1$, leading to identical solutions for (3.9). Thus, the corresponding energy levels are two-fold degenerate. (This only happens for $|\gamma| \geq|\gamma|_{\text {min }}+1$ i.e. for $j \geq q+1 / 2)$.

This can also be understood by noting that, for $\lambda= \pm q$, the spin dependence drops out in one of the $\gamma^{5}$-sectors. For $\lambda=q$, e.g., the Hamiltonian (1.1) reduces to

$$
H=\left(\begin{array}{cc}
H_{1} & \\
& H_{0}
\end{array}\right)=\frac{1}{2}\left(\begin{array}{ll}
\pi^{2}-2 q \frac{\boldsymbol{\sigma} \cdot \hat{\mathbf{r}}}{r^{2}}+\frac{q^{2}}{r^{2}} & \\
& \pi^{2}+\frac{q^{2}}{r^{2}}
\end{array}\right),
$$


i.e. $H_{0}$ describes a spin 0 particle, while $H_{1}$ corresponds to a particle with anomalous gyromagnetic ratio 4. The system admits hence an extra $o(3)$ symmetry, generated by the spin vectors

$$
\begin{array}{cc}
\mathbf{S}_{0}=\frac{1}{2} \boldsymbol{\sigma} & \mathbf{S}_{1}=U^{\dagger} \mathbf{S}_{0} U \\
\text { for } H_{0} & \text { for } H_{1},
\end{array}
$$

where $U=Q / \sqrt{H_{1}}$ and $U^{-1}=U^{\dagger}=1 / \sqrt{H_{1}} Q^{\dagger}$ are the unitary transformations which intertwine the non-zero-energy parts of the chiral sectors.

Each of the partner Hamiltonians $H_{1}$ and $H_{0}$ in $(1.1)$ have a non-relativistic conformal $o(2,1)$ symmetry [7] which combines, together with $\not D$ and $-i \gamma^{5} \not D$, into an $\operatorname{osp}(2,1)$ superalgebra [5]. Since the Hamiltonian (2.9) is essentially the same as for a free particle, the question arises whether the $\operatorname{osp}(2,1)$ likely extends into the super-Schrödinger algebra [8].

For further results the Reader is referred to [9].

\section{References}

[1] L. C. Biedenharn, Phys. Rev. 126, 845 (1962); L. C. Biedenharn and N. V. V. Swamy, Phys. Rev. 5B, 1353 (1964); M. Berrondo and H. V. McIntosh, Journ. Math. Phys. 11, 125 (1970).

[2] G. Temple, The General Principles of Quantum Mechanics, New York: Methuen (1948); P. C. Martin and R. J. Glauber, Phys. Rev. 109, 1307 (1958).

[3] F. Bloore and P. A. Horváthy, Journ. Math. Phys. 33, 1869 (1992).

[4] E. D'Hoker and L. Vinet, Phys. Rev. Lett. 55, 1043 (1986); E. D'Hoker, V. A. Kostelecky and L. Vinet, in Dynamical Groups and Spectrum Generating Algebras, p. 339-367, World Scientific, Singapore (1988).

[5] E. D'Hoker and L. Vinet, Comm. Math. Phys. 97, 391-427 (1985).

[6] Y. Kazama, C. N. Yang and A. S. Goldhaber, Phys. Rev. D15, 2287 (1977); Y. Kazama and C. N. Yang, Phys. Rev. D15, 2300 (1977).

[7] R. Jackiw, Ann. Phys. (N.Y.) 129, 183 (1980).

[8] J. P. Gauntlett, J. Gomis and P. K. Townsend, Phys. Lett. 248B, 288 (1990); C. Duval and P. A. Horváthy, Journ. Math. Phys. 35, 2516 (1994).

[9] P. A. Horváthy, A. J. Macfarlane and J.-W. van Holten, Phys. Lett. B 486, 346-352 (2000). 